\section{Spectrum of latent tuberculosis - existing tests cannot resolve the underlying phenotypes}

\section{Madhukar Pai}

In their recent Review (The spectrum of latent tuberculosis: rethinking the biology and intervention strategies. Nature Rev. Microbiol. 7, 845-855 (2009)) $)^{1}$, Barry and colleagues argue that Mycobacterium tuberculosis infection may be better viewed as a continuous nity to active infection and clinical disease ${ }^{1}$. They suggest that treatment of M. tuberculosis infection might be most effective when targeted towards the part of the spectrum that corresponds to those individuals who are at the highest risk of progression to disease. These suggestions are reasonable, and literature on interferon- $\gamma(\mathrm{IFN} \gamma)$ release assays (IGRAs) adds some support to these arguments.

Although I agree with the recommendation to give up the binary classification of latent versus active tuberculosis (TB), we are still left with a huge diagnostic challenge: none of the current tests for latent TB separate out the various possible underlying subgroups (or phenotypes) in the spectrum. The tuberculin skin test (TST) is likely to be positive in all of the proposed stages of the spectrum, with the possible exception of the phase in spectrum, extending from sterilizing immu- which infection is eliminated without priming of antigen-specific T cells. IGRAs are also likely to follow the same pattern. Thus, none of the existing tests can accurately identify the subgroup that is at risk of progression to disease. This is borne out by the sobering fact that IGRAs have been shown to have only a modest predictive ability, and several studies show similar (and rather low) rates of progression among individuals with positive TST and IGRA results ${ }^{2-6}$. For example, the incidence rate of active disease in TB contacts with positive IGRA results was 9.2 per 1000 person-years (PY) in the Gambia $^{2}, 20.5$ per 1000 PY in Turkey ${ }^{4}$ and 7.7 per $1000 \mathrm{PY}$ in Colombia ${ }^{6}$. These data suggest that even among IGRA-positive exposed individuals in TB-endemic settings, the rates of disease progression are low.

A majority of patients with a positive IGRA (or TST) will not progress to active $\mathrm{TB}$, and these individuals presumably do not need preventive therapy. This could be, in part, because IFN $\gamma$ alone might not be sufficient as a biomarker for disease progression, probably because the antigen-specific IFN $\gamma$ response is switched on in almost all stages

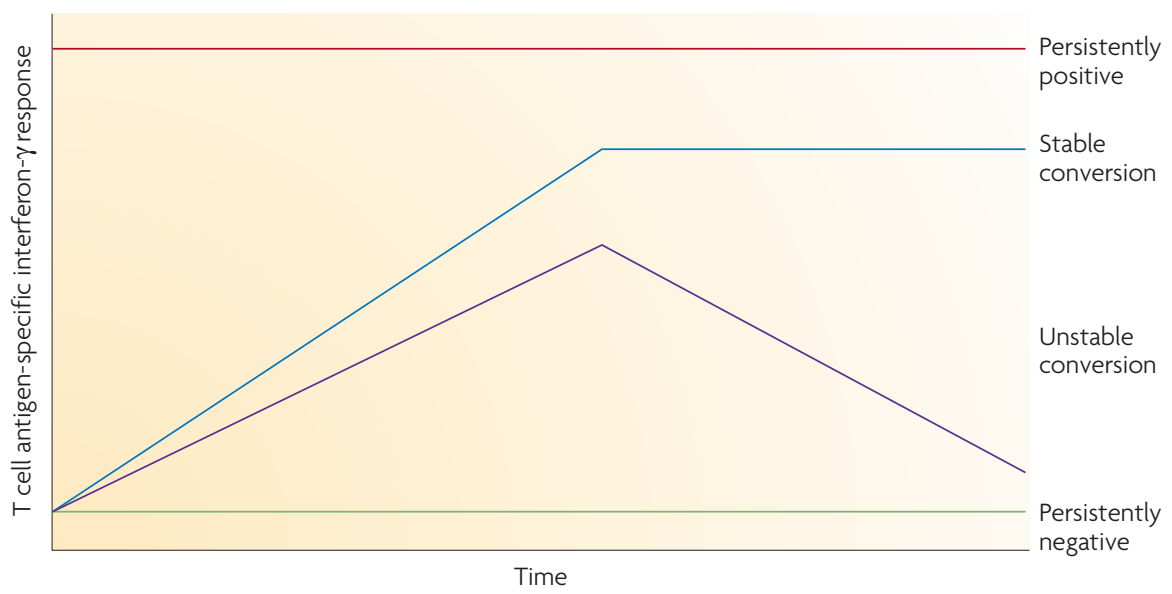

Figure 1 Serial testing with antigen-specific T cell interferon- $\gamma$ release assays reveals underlying phenotypes that are unlikely to have the same prognosis. The persistently positive pattern is seen in individuals who are repeatedly interferon- $\gamma$ release assay (IGRA)-positive for a long time. Unstable conversion refers to individuals who convert their IGRA result from negative to positive and then revert again to negativity. Stable conversion refers to individuals who convert their IGRA result and stay converted, at least in the short term. Persistently negative refers to individuals who stay repeatedly IGRA-negative for a long time. of the TB spectrum and because a positive IGRA (or TST) result does not necessarily detect live bacilli ${ }^{7}$. Furthermore, a single TST or IGRA result cannot resolve the underlying phenotypes, because they do not capture information about when infection occurred and whether the infection was fully, partly or not at all eliminated by the host. Therefore, a single TST or IGRA result may never attain high predictive or prognostic ability.

Although IGRAs may not be highly predictive, the fact that these assays can be repeated without sensitization and boosting is an advantage, because they allow us to explore the dynamics of TB infection. Repeated tuberculin tests are rarely carried out because of the belief that, once positive, a TST is likely to remain positive and, therefore, not clinically useful; even when tests are repeated, the results are hard to interpret. Serial IGRA testing shows that IGRAs are highly dynamic, with high rates of conversions and reversions $^{8-14}$. Although some of the dynamic changes may be due to test reproducibility ${ }^{13,15}$, some of the kinetics might signal the underlying transitions that occur in the TB spectrum. For example, rapid reversion of a positive IGRA after exposure is well documented ${ }^{10}$ and could imply that infection was eliminated in association with transient $\mathrm{T}$ cell priming.

On the basis of the research that I and others have carried out ${ }^{8-14}$, I believe that serial IGRA testing can reveal interesting underlying phenotypes that have different histories and trajectories (FIG. 1). Some individuals convert their IGRA result from negative to positive and then revert again. Others convert their IGRA result and stay converted, at least in the short term. Certain individuals are repeatedly IGRA-positive for a long time, and others remain IGRA-negative for years, despite ongoing TB exposure. Although no data exist to link these trajectories of test results with disease progression, it is inconceivable that all of these phenotypes will have the same prognosis. Intuitively, those who are 'persistently negative' should have the best prognosis. It likely that those who are 'persistently positive' for a long period will have a better prognosis than those who recently converted and stayed positive ('stable conversions'). Those with a transiently positive IGRA result ('unstable conversions') may have a low likelihood of disease progression, possibly similar to the persistently negative group. Without serial testing, the underlying phenotypes are not separable, and this will undermine the predictive value of a single test result. 


\section{CORRESPONDENCE}

Can the predictive value of IGRAs be enhanced by using the quantitative IFN $\gamma$ response? Are those infected individuals with strongly elevated IFN $\gamma$ levels most likely to progress to active disease? If yes, a second cut-off, higher than the diagnostic cut-off, could be used to identify the subgroup that can benefit from preventive therapy. This hypothesis has been proposed ${ }^{16}$ and is supported by limited evidence $^{6}$, but again a single test showing an elevated IFN $\gamma$ response does not provide information on when the response became elevated (whether this was recently or in the past) or whether it has stayed elevated for a long time (whether it has been persistently elevated or not). In summary, current latent $\mathrm{TB}$ tests cannot resolve the latent TB spectrum, especially with one-time testing. Without more predictive biomarkers or combinations of biomarkers, or novel serial testing strategies, we may not be able to translate biological advances into clinical interventions that can eliminate TB.
Madhukar Pai is at the Department of Epidemiology \& Biostatistics, McGill University, 1020 Pine Avenue West, Montreal, OC H3A 1A2, Canada and at the Respiratory Epidemiology \& Clinical Research Unit, Montreal Chest Institute, Montreal, H2X 2P4, Canada.

e-mail:madhukar.pai@mcgill.ca doi:10.1038/nrmicro2236-c1 Published online 19 January 2010

1. Barry, C. E. 3rd et al. The spectrum of latent tuberculosis: rethinking the biology and intervention strategies. Nature Rev. Microbiol. 7, 845-855 (2009).

2. Hill, P. C. et al. Incidence of tuberculosis and the predictive value of ELISPOT and Mantoux tests in Gambian case contacts. PLOS ONE 3, e 1379 (2008).

3. Diel, R., Loddenkemper, R., Meywald-Walter, K., Niemann, S. \& Nienhaus, A. Predictive value of a whole blood IFN- $\gamma$ assay for the development of active tuberculosis disease after recent infection with Mycobacterium tuberculosis. Am. J. Respir. Crit. Care Med. 177, 1164-1170 (2008).

4. Bakir, M. et al. Prognostic value of a T-cell-based, interferon-gamma biomarker in children with tuberculosis contact. Ann. Intern. Med. 149, 777-787 (2008).

5. Kik, S. V. et al. Predictive value for progression to tuberculosis by IGRA and TST in immigrant contacts. Eur. Respir. J. 19 Oct 2009 (doi:10.1183/09031936 .00098509).

6. del Corral, H. et al. IFN $\gamma$ response to Mycobacterium tuberculosis, risk of infection and disease in household contacts of tuberculosis patients in Colombia. PLOS ONE 4, e8257 (2009)

7. Mack, U. et al. LTBI: latent tuberculosis infection or lasting immune responses to $M$. tuberculosis?
A TBNET consensus statement. Eur. Respir. J. 33, 956-973 (2009).

8. Pai, M. et al. Serial testing of health care workers for tuberculosis using interferon- $\gamma$ assay. Am. J. Respir. Crit. Care Med. 174, 349-355 (2006).

9. Pai, M. et al. T-cell assay conversions and reversions among household contacts of tuberculosis patients in rural India. Int. J. Tuberc. Lung Dis. 13, 84-92 (2009).

10. Hill, P. C. et al. Longitudinal assessment of an ELISPOT test for Mycobacterium tuberculosis infection. PLOS Med. 4, e192 (2007).

11. Ewer, K. et al. Dynamic antigen-specific T-cell responses after point-source exposure to Mycobacterium tuberculosis. Am. J. Respir. Crit. Care Med. 174 831-839 (2006).

12. Yoshiyama, T., Harada, N., Higuchi, K., Nakajima, Y. \& Ogata, $\mathrm{H}$. Estimation of incidence of tuberculosis infection in health-care workers using repeated interferon- $\gamma$ assays. Epidemiol. Infect. 137, 1-8 (2009).

13. van Zyl-Smit, R. N. et al. Within-subject variability and boosting of T-cell interferon- $\gamma$ responses after tuberculin skin testing. Am. J. Respir. Crit. Care Med. 180, 49-58 (2009).

14. Pai, M. \& O’Brien, R. Serial testing for tuberculosis: can we make sense of $\mathrm{T}$ cell assay conversions and reversions? PLoS Med. 4, e208 (2007).

15. van Zyl-Smit, R. N., Zwerling, A., Dheda, K. \& Pai M. Within-subject variability of interferon-g assay results for tuberculosis and boosting effect of tuberculin skin testing: a systematic review. PLOS ONE 4, e8517 (2009).

16. Andersen, P., Doherty, T. M., Pai, M. \& Weldingh, K. The prognosis of latent tuberculosis: can disease be predicted? Trends Mol. Med. 13, 175-182 (2007).

Competing interests statement

The author declares no competing financial interests. 